\title{
PRODUTOS DA AGRICULTURA FAMILIAR (AF) NOS ESTADOS DO RIO DE JANEIRO E ESPÍRITO SANTO: APLICAÇÃO DA VERBA DO PROGRAMA NACIONAL DE ALIMENTAÇÃO ESCOLAR (PNAE)
}

\author{
Édira Castello Branco de Andrade Gonçalves ${ }^{1}$ \\ Amauri Corrêa Gama ${ }^{2}$ \\ Tatiana de Souza Medina ${ }^{3}$
}

\begin{abstract}
RESUMO
É estabelecido, que no mínimo $30 \%$ dos valores repassados para a alimentação escolar, deve ser aplicado na aquisição de produtos oriundos da agricultura familiar $(\mathrm{AF})$. Com intuito de identificar os agricultores familiares brasileiros criou-se a Declaração de Aptidão ao Programa Nacional de Fortalecimento da Agricultura Familiar (DAP), favorecendo na comercialização e participação na oferta da alimentação escolar. O objetivo deste estudo é avaliar a aplicação mínima do PNAE na aquisição de produtos da AF nos estados do Rio de Janeiro e Espírito Santo. Foi feito levantamento da aplicação do PNAE nas regiões intermediárias dos estados citados (2011-2017). Percebe-se predominância absoluta de DAP física em relação a DAP jurídica em ambos os estados, não garantindo maior participação desses integrantes com DAP física na repartição do montante de valores relativos à aquisição de seus produtos da agricultura familiar, conforme dispõe as normativas do PNAE. Incentivar a organização administrativa dos agricultores familiares, pode favorecer na aplicação adequada dos recursos e, consequentemente fortalecendo este setor agrícola.
\end{abstract}

Palavras-chave: agricultura familiar, política pública alimentação escolar.

\section{FAMILY AGRICULTURE (AF) PRODUCTS IN RIO DE JANEIRO AND ESPÍRITO SANTO STATES: NATIONAL SCHOOL FOOD PROGRAM (PNAE) APPLICATION}

\footnotetext{
ABSTRACT

It is established by law that at least $30 \%$ of the amounts passed on for school feeding in the municipality's education network must be applied in the acquisition of products from familiar agriculture. In order to identify Brazilian familiar farmers, the Declaration of Aptitude for the National Program for Strengthening Familiar Agriculture (DAP) has been created, which has been replaced by the National Familiar Agriculture Registry

${ }^{1}$ Graduação em Nutrição (UNIRIO) e Farmácia (UFF). Mestrado e Doutorado em Química analítica (PUC/RJ). Professor do Programa de Pós-graduação em Alimentos e Nutrição (PPGAN/UNIRIO). E-mail: ediracba.analisedealimentos@unirio.br

${ }^{2}$ Graduação em Museologia e Patrimônio (UNIRIO). Mestrado em Museologia e Patrimônio (UNIRIO). E-mail: amauricorreinha@gmail.com

3 Graduação em Nutrição (UNIRIO). Mestrado em Alimentos e Nutrição (UNIRIO). E-mail: tatiana_medina1@hotmail.com
} 
(CAF). This study aimed to evaluate the minimum application of PNAE in the purchase of AF products, in the states of Rio de Janeiro and Espírito Santo (2011-2017). Absolute predominance of physical DAP in relation to legal DAP in both states, but this not guarantee of greater participation of these members in the distribution of the amount of values related to the acquisition of their products from familiar agriculture, according to the PNAE regulations. Encouraging the administrative organization of familiar farmers can favor the proper application of PNAE resources and, consequently, strengthen this agricultural sector.

Keywords: family pharming, public policy school feeding.

\section{INTRODUÇÃO}

De acordo com a Lei no11326 de 2006, considera-se agricultor familiar e empreendedor familiar rural aqueles que praticam atividades no meio rural, atendendo, simultaneamente, aos seguintes requisitos: não deter, a qualquer título, área maior do que 4 (quatro) módulos fiscais; utilizar predominantemente mão-de-obra da própria família nas atividades econômicas do seu estabelecimento ou empreendimento; ter percentual mínimo da renda familiar originada de atividades econômicas do seu estabelecimento ou empreendimento, na forma definida pelo Poder Executivo e dirigir seu estabelecimento ou empreendimento com sua família (BRASIL, 2006)

Com o intuito de identificar os agricultores familiares brasileiros, em 1995, através da Resolução 2.191 do Conselho Monetário Nacional (CMN), criou-se a Declaração de Aptidão ao Programa Nacional de Fortalecimento da Agricultura Familiar (DAP). A DAP é o documento de identificação da agricultura familiar e pode ser obtida tanto pelos agricultores familiares (pessoa física), quanto pelo empreendimento familiar rural como associações, cooperativas, agroindústrias, ou seja, pessoa jurídica. A DAP é apresentada às instituições financeiras como forma de obtenção do crédito rural, com taxa de juros diferenciada e destinada ao fortalecimento da agricultura familiar. A campanha "Agricultura Familiar na Raiz" veiculada por 2 meses, em 2018, pela Secretaria Especial de Agricultura Familiar e do Desenvolvimento Agrário (SEAD) apresentou como primeira política a DAP, que, hoje, além de porta de entrada para o crédito, é requisito para obter acesso a outras 14 políticas da Secretaria Especial de Agricultura Familiar e do Desenvolvimento Agrário (SEAD). (BRASIL, 2009, 2017, 2018a)

O Decreto no 9.064 de 2017 dispõe sobre a Unidade Familiar de Produção Agrária (UFPA), instituindo o Cadastro Nacional da Agricultura Familiar (CAF), além de regulamentar a Lei $n^{0}$ 11.326, de 24 de julho de 2006. Considerando a última modificação no site do Ministério da Agricultura, Pecuária e Abastecimento, 20/11/2019, ainda não estão estabelecidas as condições e prazos para a transição $\mathrm{DAP} / \mathrm{CAF}$, e estando, serão publicadas e divulgadas pela Secretaria de Agricultura Familiar e Cooperativismo. Assim, entrando o CAF em vigor, este substituirá a DAP e funcionará como uma identidade por trazer características mais específicas sobre os agricultores. De acordo com o CAF, cada beneficiário terá direito a uma "carteirinha" de agricultor familiar, o que possibilitará atender de forma mais rápida este agricultor, bem como fornecer aos órgãos desenvolvedores das políticas públicas informações mais detalhadas e seguras sobre este setor (BRASIL, 2006, 2017).

Atualmente, mais de $70 \%$ dos produtos que chegam nas mesas das famílias brasileiras vem da agricultura familiar. Essa prática influencia diretamente a economia e o crescimento do país (EMBRAPA, 2017). A aquisição da agricultura familiar para a alimentação escolar está regulamentada pela Resolução n 26 , de 17 de junho de 
2013, (atualizada pela Resolução no 04, de 2 de abril de 2015), que dispõe sobre o atendimento da alimentação escolar aos alunos da educação básica no âmbito do Programa Nacional de Alimentação Escolar (PNAE), ressaltando-se a importância da legalização para a necessidade de obter a nota fiscal para a comercialização dos produtos no mercado formal (BRASIL, 2013, 2015).

O PNAE, possibilita que diferentes esferas do governo façam a aquisição de alimentos produzidos pela agricultura familiar com dispensa de licitação (GOMES et al., 2018), favorecendo este setor, que em 2015 atendeu 41,5 milhões de estudantes da educação básica em todo o país, correspondendo a 20,3\% da população brasileira.

A origem do PNAE na década de 50 , à época da criação da Campanha da Merenda Escolar, mas apenas em 1979 foi denominado de Programa Nacional de Alimentação Escolar (PNAE). Inicialmente assistencialista, desde de 1988, passou a ser um direito de todos os estudantes da rede básica de ensino e claramente a partir da Resolução CD/FNDE n 26/2013 atua na valorização da produção local de alimentos, agricultores familiares e suas associações e cooperativas.(GOMES et al., 2018; PEDRAZA et al., 2018).

A Lei no11.947 de 2009 determina que seja utilizado no mínimo 30\% dos valores repassados para a alimentação escolar na rede de ensino dos municípios, diretamente com compras de gêneros alimentícios da agricultura familiar e do empreendedor familiar rural, ou de suas organizações, priorizando-se os assentamentos da reforma agrária, as comunidades tradicionais indígenas e as comunidades quilombolas. A lei dispõe que a alimentação saudável e adequada compreende o uso de alimentos variados, seguros, que respeitem a cultura, as tradições e os hábitos alimentares saudáveis, bem como determina o apoio ao desenvolvimento sustentável, com incentivos para a aquisição de gêneros alimentícios diversificados, sazonais, produzidos em âmbito local e pela agricultura familiar (BRASIL, 2009).

Considerando que não é aplicada a verba do PNAE, como estabelece a legislação, este estudo teve como objetivo avaliar esta realidade nos estados do Rio de Janeiro e Espírito Santo (2011-2017).

\section{MATERIAL E MÉTODOS}

Como critérios metodológicos optou-se por realizar 2 etapas envolvendo captação de dados, considerando a aplicação da verba do PNAE e potencial de produção agrícola dos estados estudados, conforme segue:

\section{Identificação da aplicação verba PNAE na agricultura familiar}

Foi feito um levantamento da aplicação da verba do PNAE para aquisição de produtos oriundos da agricultura familiar por regiões dos estados do Rio de Janeiro e Espírito Santo, sendo estas regiões subdivididas em Regiões Intermediárias de acordo com o IBGE (IBGE, 2017).

\section{Identificação do potencial de produção da agricultura familiar dos municípios}

Cada Região Intermediária foi avaliada quanto ao potencial de produção de produtos da agricultura familiar, a partir da quantidade de DAP jurídica e física, considerando que está em processo de transição a alteração DAP/CAF. A consulta foi feita no Sistema DAP da Secretaria Especial da Agricultura Familiar e do Desenvolvimento Agrário (BRASIL, 2018a). 


\section{RESULTADOS E DISCUSSÃO}

Os dados disponibilizados nas bases utilizadas, IBGE e sistema DAP, conforme descrito na metodologia, para a pesquisa correspondem à totalidade dos municípios dos estados do Rio de Janeiro e Espírito Santo, com especial atenção para aqueles que são contemplados com recursos para aplicação na aquisição de produtos oriundos da agricultura familiar local na alimentação escolar, e desta forma, foram considerados 53 municípios do estado do Espírito Santo e 45 municípios do estado do Rio de Janeiro, todos participantes da política pública de transferência desses recursos.

Observando a figura 1, verifica-se que o número de DAPs, física e jurídica, além da participação em cooperativas no Espírito Santo é 5 vezes superior ao estado do Rio de Janeiro. Analisando cada estado, o número de DAP física é 13 vezes maior que o número de DAP jurídica no Espírito Santo; no estado do Rio de Janeiro há predominância também de DAP física, chegando ao total de mais de 20 mil integrantes, praticamente dez vezes maior do que o total de integrantes com DAP jurídica.

Figura 1 - Comparação do número de cooperativas, integrantes com DAP jurídica, DAP física e número total de integrantes nas cooperativas nos estados do Espírito Santo (A) e no Rio de Janeiro (B).
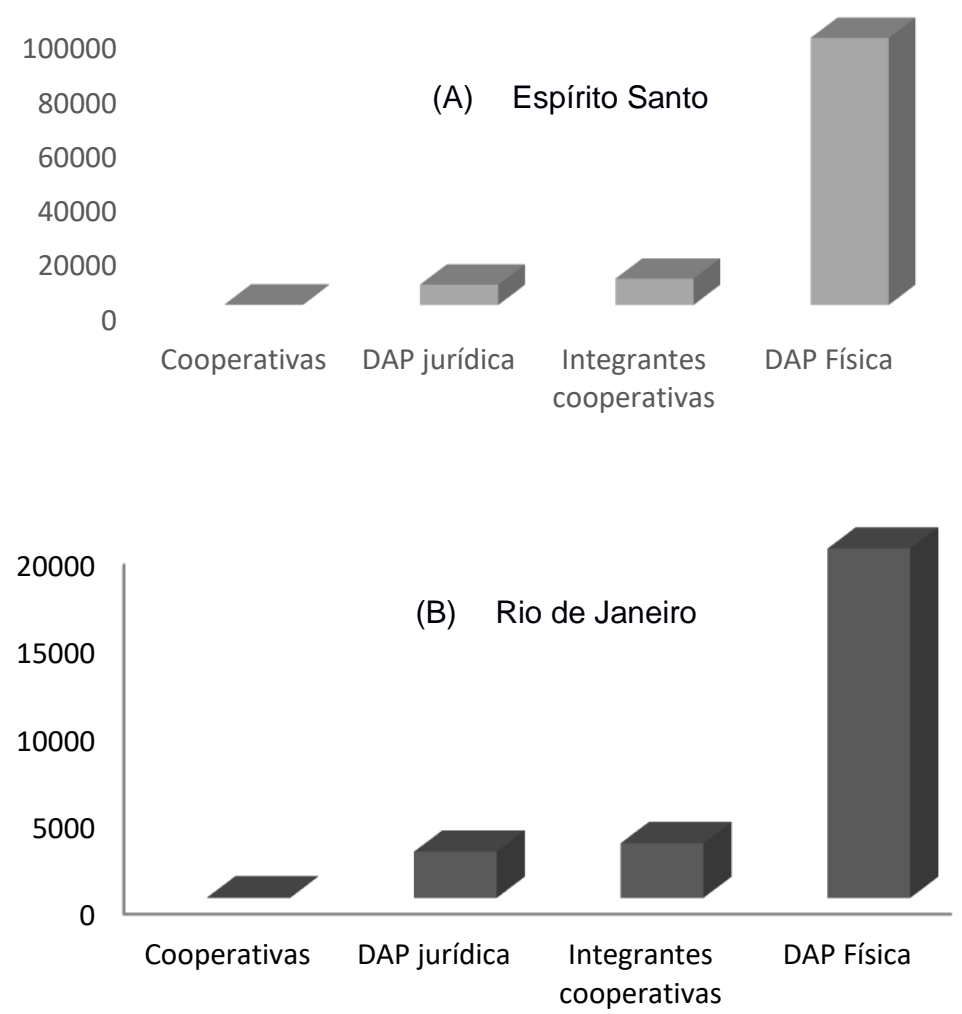

Fonte: Sistema DAP da Secretaria Especial da Agricultura Familiar e do Desenvolvimento Agrário (BRASIL, 2018a). 
No Espírito Santo, as regiões geográficas intermediárias (IBGE, 2017) são representadas por: Vitória (V), São Mateus (SM), Colatina (C) e Cachoeiro de Itapemirim $(\mathrm{Cl})$ Considerando que o total de integrantes com DAP física no estado é 97.999 (figura 1) e este se divide em 4 regiões, como os limítrofes mínimo e máximo associados as regiões $\mathrm{Cl}(21.900)$ e V (23.728), representando 47\% do total do estado, observa-se boa distribuição da AF não havendo discrepâncias nas diferentes regiões.

No estado do Rio de Janeiro, são 5 regiões geográficas intermediárias (IBGE, 2017): Rio de Janeiro (RJ), Volta Redonda e Barra Mansa (VRBM), Petrópolis (P), Campos dos Goytacazes (CG) e Macaé - Rio das Ostras - Cabo Frio (MRC). Os integrantes com DAP jurídica representam pouco mais de 2,6 mil agricultores familiares (Figura 1), sendo as regiões $P$ e VRBM limítrofes máxima e mínima, respectivamente 686 e 234 integrantes com DAP jurídica, representando $35 \%$ do total. Já os integrantes com DAP física deste estado, pouco mais de 20 mil (figura 1), apresentam limítrofes representados pelas regiões RJ (mínimo) com 1090 e P e CG (máximo), ambas acima de 7 mil. Observa-se que no estado do Rio de Janeiro, ao contrário do estado do Espírito Santo, não há uniformidade da AF nas diferentes regiões.

Foi feita a análise do número de municípios por regiões intermediárias dos estados do Espírito Santo e Rio de Janeiro que aplicam valor inferior a $30 \%$ dos repasses dos recursos do PNAE para aquisição de produtos da agricultura familiar (IBGE, 2017), figura 2.

Segundo Rodrigues et al., 2017, em 2010, 47,4\% dos municípios brasileiros fizeram aquisição via PNAE, sendo que o porcentual de compra foi, em média, de $22,7 \%$ do repasse/FNDE, abaixo do mínimo exigido (30\%) pela Lei de 2009 (RODRIGUES et al., 2017). De acordo com Machado et al. (2018), o estado do Espírito Santo destaca-se na região Sudeste como o de maior percentual de realização de compra, e dentre os $41,5 \%$ dos municípios do sudeste com gastos adequados à legislação, o Espírito Santo é que apresenta a maior aplicação da verba, com 63,8\% de seus municípios aplicando acima dos $30 \%$ dos recursos na compra da agricultura familiar (MACHADO et al., 2018).

De acordo com os dados do FNDE de 2018, dos 53 municípios analisados do estado do Espírito Santo, 41,5\% não aplicam 30\% da verba do PNAE para aquisição de produtos da agricultura familiar. Dos 10 municípios da região Colatina, $60 \%$ não cumpriram as exigências dos 30\% com aquisição de produtos da agricultura familiar. No caso da região $\mathrm{V}$, dos 14 municípios, $50 \%$ não atingiram o mínimo exigido por lei. A região SM, com 12 municípios, possui $42 \%$ dos municípios que não cumprem a exigência. Por fim, a região $\mathrm{Cl}$ representada por 24 municípios, é a que menos teve problemas com investimentos e somente $27 \%$ não cumpriram as exigências legais. A Região $C$ foi a que menos aplicou a verba, pois dos seus 10 municípios, $60 \%$ não atenderam à legislação. Em contrapartida, o município que mais aplicou a verba, superando os $30 \%$ exigidos pela legislação, foi Alegre, da Região $\mathrm{Cl}$, com $96 \%$ aplicados na aquisição de produtos da agricultura familiar. Este município, vem realizando a aquisição de alimentos da agricultura familiar pelo PNAE desde 2010, mas a partir de 2014 vem aumentando tanto na diversidade quanto na quantidade de alimentos, e as ações coletivas dos agricultores familiares foram decisivas para este processo, potencializando a interligação entre agricultura familiar local e alimentação escolar (RODRIGUES et al., 2017).

O Espírito Santo, conforme já mencionado, é um dos estados da região Sudeste que mais se adequa na aplicação dos recursos na compra da agricultura familiar (MACHADO et al., 2018), mas, não há uniformidade de ações junto ao PNAE 
das diferentes regiões do estado, apresentando regiões com até $73 \%(\mathrm{Cl})$ de adequação e outras $40 \%$ (C). A modalidade Compra Institucional que engloba o PNAE necessita de amplo interesse governamental, além dos produtores, sendo fundamental a criação de redes de associações produtivas, agroalimentares alternativas e programas governamentais nas diferentes esferas (MARTINELLI; CAVALLI, 2019).

Figura 2 - Percentual (\%) de municípios por regiões intermediárias dos estados do Espírito Santo (A) [Vitória (V), São Mateus (SM), Colatina (C) e Cachoeiro de Itapemirim (Cl)] e Rio de Janeiro (B) [Rio de Janeiro (RJ), Volta Redonda e Barra Mansa (VRBM), Petrópolis (P), Campos dos Goytacazes (CG) e Macaé - Rio das Ostras - Cabo Frio (MRC)] que aplicam valor menor que $30 \%$ do Programa Nacional de Alimentação Escolar (PNAE) para aquisição de produtos oriundos da agricultura familiar.

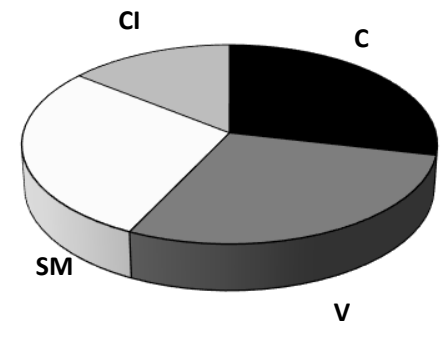

(A) Espírito Santo

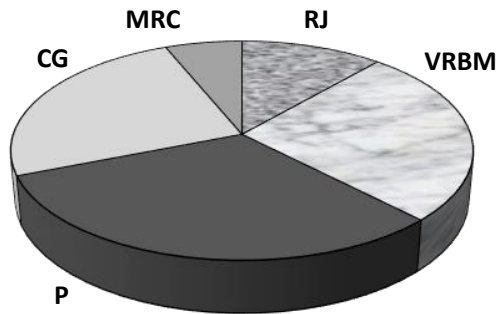

(B) Rio de Janeiro

O Estado do Rio de Janeiro é o de menor índice de compra, 30\%, ou seja, $70 \%$ dos municípios deste estado não cumpriram a legislação quanto a aplicação de recursos da agricultura familiar no PNAE (MACHADO et al., 2018). Em planilha referente aos repasses do Ministério da Educação para o exercício de 2016, e que possui dados atualizados até 2018 (BRASIL, 2018b), dos 92 municípios do Estado do Rio de Janeiro, 45,7\% receberam recursos aptos a serem aplicados com a alimentação escolar utilizando o mínimo de $30 \%$ vinculados legalmente para compras de alimentos da agricultura familiar local. No entanto, 59,5\% desses municípios que receberam recursos não cumpriram o que a lei exige, e entre esses que não cumpriram a lei, 16,7\% aplicaram zero recursos na aquisição de alimentos da agricultura familiar.

Na sequência da análise do estado do Rio de Janeiro, a região RJ representa $11 \%$ que não cumpriram a aplicação mínima de 30\%. Dos 26 municípios desta região, $15,4 \%$ não atingiram o mínimo exigido e 3,8\% aplicaram zero recursos. VRBM, dos 17 municípios, $23,5 \%$ não atingiram o mínimo exigido por lei, e 11,8\% aplicaram zero recursos. Esta macrorregião representa $26 \%$ do total de todas as macrorregiões do estado que não cumpriram a legislação de aplicação mínima. A região $P$, com 19 municípios, representa $31 \%$ do total das macrorregiões do estado que estão irregulares na aplicação, com $31,6 \%$ dos municípios que não cumprem a exigência dos $30 \%$, e $10,5 \%$ aplicaram zero recursos. A região CG representa $42,1 \%$ do total das macrorregiões do estado; de seus 18 municípios, 31\% aplicaram abaixo de $30 \%$ exigidos na lei. A região MRC possui 12 municípios e representa $8,3 \%$ do total das 
macrorregiões que não cumpriram as exigências dos $30 \%$ com aquisição de produtos da agricultura familiar, com $8,3 \%$ dos municípios não cumprindo o exigido em lei.

O único município, do Estado do Rio de Janeiro, que aplicou a totalidade de $100 \%$ de todo o recurso transferido para a educação municipal - não somente os $30 \%$ exigidos por lei, mas a totalidade dos repasses transferidos pelo Governo Federal para o município com a aquisição de produtos da agricultura familiar, foi Macaé, da Região Intermediária MRC, acompanhado de perto por Bom Jardim, da Região Intermediária $\mathrm{P}$, que aplicou mais de $97 \%$ dos recursos.

A figura 3 apresenta a distribuição dos integrantes com DAP jurídica por regiões intermediárias. O estado do Espírito Santo apresenta distribuição similar dos representantes de DAP jurídica, em 3 regiões (C; $V$ e $S M)$, apenas a região $\mathrm{Cl}$ diverge, representando $33 \%$ do total do estado, enquanto as demais, variam de 21 a $24 \%$.

O estado do Rio de Janeiro apresenta distribuição não uniforme dos integrantes com DAP jurídica. A região RJ é a que mais possui integrantes (45\%), e as regiões de CG e VRBM são as que possuem menos integrantes (9\%).

As regiões intermediárias que comportam as capitais de ambos os estados apresentam diferenças marcantes em relação ao quantitativo de DAP. Na região V (ES), integrantes com DAP física são o dobro quando comparados com a região $R J$ $(R J)$. Já para DAP jurídica, a região RJ (RJ) apresenta o dobro de integrantes, quando comparados com a região $\mathrm{V}(\mathrm{ES})$.

Figura 3 - Percentual (\%) DAP jurídica das regiões intermediárias dos estados do Espírito Santo (A) [Vitória (V), São Mateus (SM), Colatina (C) e Cachoeiro de Itapemirim (Cl)] e Rio de Janeiro (B) [Rio de Janeiro (RJ), Volta Redonda e Barra Mansa (VRBM), Petrópolis (P), Campos dos Goytacazes (CG) e Macaé - Rio das Ostras - Cabo Frio (MRC)] que aplicam valor menor que 30\% do Programa Nacional de Alimentação Escolar (PNAE) para aquisição de produtos oriundos da agricultura familiar.

\section{(A) Espírito Santo}

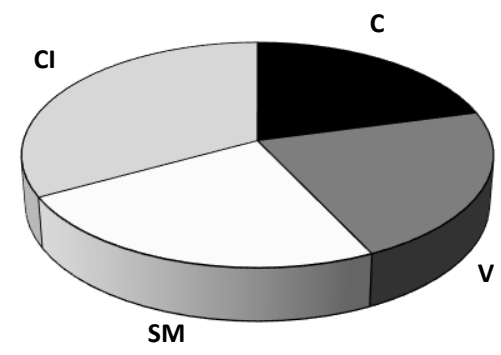

(B) Rio de Janeiro

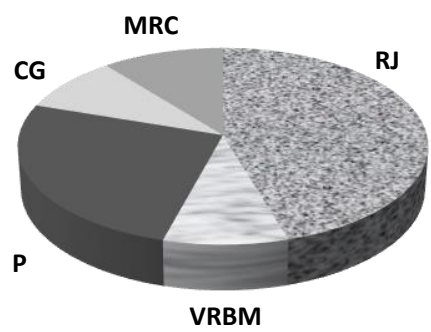

Em relação a aplicação dos recursos abaixo dos 30\% exigidos por lei, os dois estados se diferenciam bastante, com mais equilíbrio para o estado do Espírito Santo, em que ocorreram índices de aplicação dos recursos relativamente equilibrados, entre 22 e $29 \%$. Já no estado do Rio de Janeiro, a região da capital do estado, RJ, obteve índices relativamente melhores, com apenas $11 \%$ de seus municípios não cumprindo o mínimo de $30 \%$ exigidos por lei.

Estudo recente, realizado por Machado et. al. 2018, correlacionam menor aplicação dos recursos do PNAE na aquisição de produtos da agricultura familiar com 
a ausência de nutricionistas como responsável técnico da alimentação escolar, indicando que o estado do Rio de Janeiro é o que possui menor presença deste profissional (MACHADO et al., 2018).

Percebe-se pelos números que há predominância absoluta de DAP física em relação a DAP jurídica em ambos os estados citados, mas isso não significa a garantia de maior participação desses na repartição do montante de valores relativos à aquisição de seus produtos da agricultura familiar para compor o cardápio da alimentação no ensino público, conforme dispõe as normativas do PNAE.

A Portaria 129 de 2018 instituiu o selo de identificação da participação da agricultura familiar (SIPAF). De acordo com o Instituto Capixaba de Pesquisa, Assistência Técnica e Extensão Rural (Incaper), o incentivo ao selo faz parte do Projeto "Estruturação e Fortalecimento dos Setores Produtivos da Agricultura Familiar do Norte do Espírito Santo - Tecsocial". Este incentivo tem como objetivo identificar, adequar e reaplicar tecnologias sociais para fortalecer e estruturar organizações associativas de agricultores familiares e, com isso, agregar valor para comercializar a produção (BRASIL, 2018b).

Em específico e exclusivo para cooperativas, há um incentivo do Programa Nacional de Fortalecimento da Agricultura Familiar (PRONAF) chamado Cota-Parte que financia investimentos para a integralização de cotas-partes dos agricultores familiares filiados a cooperativas de produção ou para aplicação pela cooperativa em capital de giro, custeio ou investimento (BNDES, 2020)

Outra informação relevante é sobre os produtos agrícolas mais produzidos nos estados. No Espírito Santo, o produto de maior relevância é o café. Outros produtos como pimenta-do-reino, cana-de-açúcar, milho e mamão contribuem de forma significativa para retorno financeiro do estado. Os 5 maiores municípios neste quesito de acordo com os dados do INCAPER (INCAPER, 2017) são Santa Maria de Jetibá, São Mateus, Linhares, Jaguaré e Domingos Martins. Santa Maria de Jetibá e Domingos Martins estão na região V enquanto São Mateus, Linhares e Jaguaré estão na região SM que é a segunda região com mais DAP jurídica. Com estes dados, podese sugerir que a organização dos agricultores familiares em pessoa jurídica (DAP jurídica), pode ser um facilitador para incrementar a comercialização dos produtos.

Considerando que o PNAE tem como proposta respeitar, promover, proteger, facilitar e prover o Direito Humano a Alimentação Adequada (DHAA) (BOCCHI et al., 2019) (SOUZA et al., 2018), observar fragilidades na execução deste programa é impactar na qualidade da dieta de milhares de indivíduos. Ações afirmativas da academia, como as executadas pela UFPel, devem ser incentivadas visando aumentar a aquisição de produtos da AF local por mercados institucionais (SOUZA et al., 2018).

Promover uma alimentação saudável é uma das metas do PNAE, assim a aquisição de alimentos da agricultura local além de incentivar a economia local ,contribui com a oferta e o consumo de alimentos saudáveis (SOARES et al., 2018a). É de extrema importância refletir sobre os meios possíveis para que possam ser promotores de uma inovação social regional e no país; neste contexto a criação de mercados institucionais na Amazônia buscou combater problemas sociais como fome e pobreza, a partir da alimentação escolar fortalecida pela agricultura familiar regional (OLIVEIRA; SOBRINHO, 2020). A organização dos agricultores familiares em associações e cooperativas não garante a participação no PNAE; estudo realizado no Rio Grande do Norte mostrou que das 2.513 organizações sociais e de produtores familiares (2.479 associações e 34 cooperativas) apenas $6 \%$ participam efetivamente dos conselhos municipais de alimentação escolar (CMAE's) e somente 1\% utilizam os recursos do PNAE para financiar seus projetos (DIAS; OLIVEIRA, 2019) 
Em 2016, a Assembleia Geral das Nações Unidas aprovou Resolução, Década da Nutrição, endossando o compromisso com a Nutrição; nesta perspectiva, múltiplos fatores devem ser considerados visando o aporte de alimentos saudáveis, produzidos com sustentabilidade e respeitando as diversidades culturais $(\mathrm{BOCCHI}$ et al., 2019). A agricultura familiar é um dos grandes pilares que podem promover a distribuição de alimentos saudáveis, atendendo as diversidades culturais e ainda impactando positivamente na sustentabilidade regional. A produção agrícola familiar é a principal atividade econômica de muitas regiões do Brasil, além de promover menor impacto ambiental (GUILLEN; COTI-ZELATI; ARAÚJO, 2020) e sendo desta forma, no mínimo se espera que os órgãos governamentais cumpram o estabelecido pelo $\mathrm{PNAE}$, e que sejam realizados estudos visando o aumento da margem mínima obrigatória de aplicação da verba do PNAE neste setor (SOARES et al., 2018b).

No que diz respeito as diversidades culturais, poucos estudos associam o regionalismo ao enfoque da Nutrição, mas é relevante o papel do nutricionista no resgate à cultura e aos hábitos alimentares, e nesta perspectiva a ação do PNAE que atende mais de 46 milhões de brasileiros é um meio de promoção do regionalismo alimentar a partir dos cardápios desenvolvidos (BOCCHI et al., 2019; GUIMARÃES et al., 2019).

Além dos entraves na aplicação do PNAE que dificultam a expansão do mercado da agricultura, importante mencionar que a atual conjuntura do regime alimentar corporativo, provoca profunda crise socioambiental. Fator relevante neste contexto são os conceitos de Segurança Alimentar e Nutricional e Soberania Alimentar gerando oportunidades para a agricultura familiar participar da construção social do mercado agroecológico (MUNOZ; NIEDERLE, 2018).

\section{CONCLUSÕES}

É importante uma análise mais técnica e detalhada da transferência e aplicação dos recursos destinados a alimentação escolar e as leis que estabelecem a aplicação na aquisição de produtos oriundos da agricultura familiar, observando correlação na distribuição ou concentração desses recursos, principalmente com prevalência para a DAP jurídica, cujos integrantes se apresentam estruturalmente melhor organizados. Por sua vez, é provável ocorrerem dificuldades burocráticas para a inserção e participação ativa de produtores da agricultura familiar identificados com DAP física, visto se mostrarem menos organizados coletivamente. O incentivo a organização administrativa dos agricultores familiares, pode favorecer na aplicação adequada dos recursos do PNAE e, consequentemente fortalecendo este setor agrícola.

\section{REFERÊNCIAS}

\section{BNDES. Pronaf - Programa Nacional de Fortalecimento da Agricultura Familiar $2020 . \quad$ Disponível em:} https://www.bndes.gov.br/wps/portal/site/home/financiamento/produto/pronaf. Acesso em: 26 jun. 2020.

BOCCHI, C. P.; MAGALHÃES, É. S.; RAHAL, L.; GENTIL, P.; GONÇALVES, R. S. The nutrition decade, the public policy for food security, and public purchases from family farming in BrazilEI Decenio de las Naciones Unidas de Acción sobre la Nutrición, la política de seguridad alimentaria y nutricional, y las compras públicas de la a.

Revista panamericana de salud publica $=$ Pan American journal of public health, v. 43, p. e84-e84, 2019. Disponível em: https://pubmed.ncbi.nlm.nih.gov/31892923. 
Acesso em: 26 jun. 2020.

BRASIL. Lei $n^{\circ} 11.326$ de 24 de julho de 2006. Diário Oficial da República Federativa do Brasil Poder executivo, [S. I.], p. 25/07/2006, p.1, 2006. Disponível em:https://legislacao.presidencia.gov.br/atos/?tipo=LEI\&numero=11326\&ano=2006\& ato=981MTRU5kMRpWTf02. Acesso em: 26 jun. 2020.

BRASIL. Lei $\mathrm{n}^{\circ} 11.947$ de 16 de junho de 2009. Diário Oficial da República Federativa do Brasil Poder executivo, [S. I.], p. 17/06/2009, P. 2, 2009. Disponível em:https://legislacao.presidencia.gov.br/atos/?tipo=LEI\&numero=11947\&ano=2009\& ato=359AzaE90dVpWTc00. Acesso em: 26 jun. 2020.

BRASIL. Resolução/CD/FNDE $n^{\circ} 26$, de 17 de junho de 2013. Diário Oficial da República Federativa do Brasil Poder executivo, [S. I.], p. 18/06/2013 | Edição: 115 | Seção: 1 | Página: 7| E, 2013. Disponível em: http://www.in.gov.br/materia//asset_publisher/Kujrw0TZC2Mb/content/id/30683767/do1-2013-06-18-resolucao-n26-de-17-de-junho-de-2013-30683763. Acesso em: 26 jun. 2020.

BRASIL. Resolução/CD/FNDE/MEC n 4, de 3 de abril de 2015. 2015. Disponível em:https://www.fnde.gov.br/index.php/acesso-a-

informacao/institucional/legislacao/item/6341-resolução-cd-fnde-mec-nº-4,-de-3-deabril-de-2015. Acesso em: 26 jun. 2020.

BRASIL. Decreto n $n^{\circ} 9.064$ de 31 de maio de 2017. Diário Oficial da União Poder Executivo, [S. I.], p. 31/05/2017, P. 11 EDIÇÃO EXTRA, 2017. Disponível em: https://legislacao.presidencia.gov.br/atos/?tipo=DEC\&numero $=9064 \& a n o=2017 \&$ ato =d88oXRE1UeZpWTd3c. Acesso em: 26 jun. 2020.

BRASIL, Casa Civil. Secretaria Especial da Agricultura Familiar e do Desenvolvimento Agrário. Sistema de Consultas DAP. 2018a. Disponível em: http://dap.mda.gov.br/. Acesso em: 26 jun. 2020.

BRASIL, Casa Civil. Portaria № 129, de 7 de março de 2018. 2018b. Disponível em: http://www.in.gov.br/materia/-

/asset_publisher/Kujrw0TZC2Mb/content/id/5763601/do1-2018-03-08-portaria-n-129de-7-de-marco-de-2018-5763597. Acesso em: 26 jun. 2020.

DIAS, T. F.; OLIVEIRA, E. F. Agricultura Familiar, Políticas Públicas e Mercados Institucionais: uma Análise Exploratória do Programa Nacional de Alimentação Escolar - PNAE no Rio Grande do Norte. HOLOS, v. 5, n. 0, p. 1-19, 2019. Disponível em: http://www2.ifrn.edu.br/ojs/index.php/HOLOS/article/view/6838. Acesso em: 26 jun. 2020.

EMBRAPA. A real contribuição da agricultura familiar no Brasil - Portal Embrapa. 2017. Disponível em: https://www.embrapa.br/agropensa/busca-de-noticias//noticia/27405640/a-real-contribuicao-da-agricultura-familiar-no-brasil. Acesso em: 26 jun. 2020.

GOMES, A. C., DEPONTI, C. M., AREND, S. C., ETGES, V. E., KARNOPP, E., SILVA, T. L. DA.; BOER, A. I. O mercado institucional da compra de alimentos da agricultura familiar - PAA E PNAE - no território do Vale do Rio Pardo, RS. Desenvolvimento Regional em debate, v. 8, n. 1, p. 4-24, 2018. Disponível em: 
http://www.periodicos.unc.br/index.php/drd/article/view/1544. Acesso em: 26 jun. 2020.

GUILLEN, J. F.; COTI-ZELATI, P. E.; ARAÚJO, D. L. A. family farming and sustainable development of agribusiness in the metropolitan region of Campinas. Revista Metropolitana de Sustentabilidade, v. 10, n. 1, p. 123-145, 2020.

GUIMARÃES, E. P. B.; MARQUES, J. M. S.; SILVA, L. L. L.; CARDOSO, C. G. L. V. Regionalismo presente nos cardápios da alimentação escolar no município de Campinorte - Goiás. Hygeia - Revista Brasileira de Geografia Médica e da Saúde, v. 15, n. 31, 2019. Disponível em: http://www.seer.ufu.br/index.php/hygeia/article/view/47097. Acesso em: 26 jun. 2020.

IBGE. Divisão Regional do Brasil | IBGE. 2017. Disponível em: https://www.ibge.gov.br/geociencias/organizacao-do-territorio/divisao-

regional/15778-divisoes-regionais-do-brasil.html?=\&t=0-que-e. Acesso em: 26 jun. 2020.

INCAPER. Publicação do Incaper apresenta dados da produção dos municípios capixabas. 2017. Disponível em: https://incaper.es.gov.br/Notícia/publicacao-doincaper-apresenta-dados-da-producao-dos-municipios-capixabas. Acesso em: 26 jun. 2020.

MACHADO, P. M. O. et al. Compra de alimentos da agricultura familiar pelo Programa Nacional de Alimentação Escolar (PNAE): estudo transversal com o universo de municípios brasileiros. Ciência \& Saúde Coletiva, v. 23, n. 12, p. 4153-4164, 2018. Disponível em: http://www.scielo.br/scielo.php?script=sci_arttext\&pid=S1413$81232018001204153 \&$ Ing=pt\&tIng=pt. Acesso em: 27 jun. 2020.

MARTINELLI, S. S.; CAVALLI, S. B. Alimentação saudável e sustentável: uma revisão narrativa sobre desafios e perspectivas. Ciência \& Saúde Coletiva, v. 24, n. 11, p. 4251-4262, $2019 . \quad$ Disponível em: http://www.scielo.br/scielo.php?script=sci_arttext\&pid=S1413-

$81232019001104251 \&$ tIng=pt. Acesso em: 27 jun. 2020.

MUNOZ, E.; NIEDERLE, P. Críticas cívicas ao regime alimentar corporativo: mercados da reforma agrária em Porto Alegre, RS. Geo Uerj, Rio de Janeiro, n.33, p.1-17, 2018. Disponível em: https://www.epublicacoes.uerj.br/index.php/geouerj/article/view/33779/28267. Acesso em: 27 jun. 2020.

OLIVEIRA, R. S.; SOBRINHO, M. V. Gestión de Políticas Públicas para el Desarrollo Local en Brasil. GICAPP Estudios/working papers, v. 7, p. 140-149, 2020. Disponível em: http://www.gigapp.org/ewp/index.php/GIGAPP-EWP/article/view/169. Acesso em: 26 jun. 2020.

PEDRAZA, D. F.; MELO, N. L. S.; SILVA, F.A.; ARAUJO, E.M.N.; Avaliação do Programa Nacional de Alimentação Escolar: revisão da literatura. Ciência \& Saúde Coletiva, [S. I.], v. 23, n. 5, p. 1551-1560, 2018. DOI: 10.1590/141381232018235.17832016. Disponível

http://www.scielo.br/scielo.php?script=sci_arttext\&pid=S1413-

$81232018000501551 \&$ Ing=pt\&tlng=pt. Acesso em: 27 jun. 2020. 
RODRIGUES, R. I; SIQUEIRA, H. M.; BIANCARDI, C. C. S.; ANDRADE, M. A. N.; VALENTE, L. M.; PAULA, L. B. Aquisição de alimentos da agricultura pelo pelo país no município de Alegre-ES. DEMETRA: Alimentação, Nutrição \& Saúde, v. 12, n. 1, p. $91-112,2017$.

SOARES, P. et al. Programa Nacional de Alimentação Escolar como promotor de Sistemas Alimentares Locais, Saudáveis e Sustentáveis: uma avaliação da execução financeira. Ciência \& Saúde Coletiva, v. 23, n. 12, p. 4189-4197, 2018a. Disponível em: $\quad$ http://www.scielo.br/scielo.php?script=sci_arttext\&pid=S1413$81232018001204189 \&$ Ing=pt\&tIng=pt. Acesso em: 26 jun. 2020.

SOARES, P.; MARTINELLI, S. S.; FABRI, R. K.; VEIROS, M. B.; DAVO-BLANES, M. C.; CAVALLI, S. B. Brazilian National School Food Program as a promoter of local, healthy and sustainable food systems: evaluating the financial implementation. Ciência \& Saúde Coletiva, v. 23, p. 4189+, 2018b. Disponível em: https://link.gale.com/apps/doc/A571680328/AONE?u=capes\&sid=AONE\&xid=5f1771 9c. Acesso em: 26 jun. 2020.

SOUZA, C. A. N. et al. Adequação nutricional e desperdício de alimentos em Centros de Educação Infantil. Ciência \& Saúde Coletiva, v. 23, n. 12, p. 4177-4188, 2018. Disponível em: http://www.scielo.br/scielo.php?script=sci_arttext\&pid=S1413$81232018001204177 \&$ Ing=pt\&tIng=pt. Acesso em: 26 jun. 2020. 